\title{
Aprendizaje Reflexivo del profesorado para atención a la diversidad. Propuestas de mejora para España y México
}

\author{
Miñán Espigares, Antonio \\ Universidad de Granada, Granada, España \\ aminan@ugr.es
}

\begin{abstract}
Juárez Romero, Claudia Amanda
Benemérita Escuela Nacional de Maestros, Ciudad de México, México amandajuarezrom.unam@gmail.com
\end{abstract}

\author{
Alcántara Santuario, Armando \\ Universidad Nacional Autónoma de México, Ciudad de México, México \\ aralsantuario@gmail.com
}

\section{Resumen}

En este trabajo se plantea potenciar el aprendizaje reflexivo en la formación de profesorado, tanto inicial como continua, en relación con el aprendizaje para avanzar en la inclusión. Se trata de una investigación cualitativa comparada, con una muestra de 50 alumnos/as en México y España. Sus observaciones en la práctica y sus opiniones acerca del conocimiento que tienen de la práctica nos ayuda a conocer los aspectos fundamentales para una adecuada formación para la diversidad. Las observaciones realizadas por estudiantes de España y México han sido comparadas, obteniendo una conclusión en forma de sugerencia para mejorar los programas formativos. Algunos de los resultados importantes del trabajo hacen referencia a la importancia de no poner límites a ningún alumno y permitir que avancen hasta donde puedan pero sin que nuestras expectativas como docentes influyan o determinen. También hemos podido comprobar que aunque la necesidad de respetar e incluir todas las culturas es común en ambos países, en España parece que predomina el alumnado procedente de otros países, mientras que en México, proceden de otros estados. En ambos casos se debe atender a las diferencias culturales o contextuales. Una de las claves de la inclusión es tratar a todos con el mismo respeto. En ambos países se debe avanzar mucho para conseguir niveles de inclusión mayores. No sólo en la Escuela sino en la sociedad.

\section{Abstract}

In this work, it is proposed to promote reflective learning in teacher training, both initial and ongoing, in relation to learning to advance in inclusion. It is a comparative qualitative research, with a sample of 50 students in Mexico and Spain. Their observations in practice and their opinions about the knowledge they have of the practice helps us to know the fundamental aspects for an adequate formation for diversity. The observations made by students from Spain and Mexico have been compared, obtaining a conclusion in the form of a suggestion to improve the training programs. Some of the important results of the work refer to the importance of not placing limits on any student and allowing them to advance as far as they can but without our expectations as teachers influencing or determining. We have also seen that although the need to respect and include all cultures is common in both countries, in Spain it seems that students from other countries predominate, while in Mexico, they come from other states. In both cases, cultural or contextual differences must be addressed. One of the keys to inclusion is to treat everyone with the same respect. In both countries, much progress must be made to achieve higher levels of inclusion. Not only in the School but insociety.

Palabras clave: formación del profesorado, atención a la diversidad, inclusión, aprendizaje reflexivo.

Keywords: teacher training, attention to diversity, inclusion, reflective learning.

\section{INTRODUCCIÓN}

En el ámbito de la docencia universitaria se está poniendo énfasis en el incremento del aprendizaje reflexivo. Desde la perspectiva del profesional reflexivo se entiende la práctica docente como un escenario donde se producen conocimientos y se concibe al profesorado como poseedor de teorías 
implícitas que constituyen un pilar muy importante para la enseñanza (Anijovich et al, 2009). Estos conocimientos y estas teorías implícitas pueden gestarse ya desde la formación inicial del profesorado. En el caso de la formación para atender la diversidad resulta especialmente importante este aprendizaje reflexivo. Este trabajo se puede aplicar también al profesorado en ejercicio.

Según Llorent y Llorent (2012), la opinión que tengan de la Educación Inclusiva los futuros maestros es determinante para la escuela. Estos autores realizan un estudio de Educación Comparada utilizando el análisis documental, entrevistas, evocaciones libres y encuestas. En este caso se comparan las opiniones de los futuros maestros y maestras de: Río de Janeiro (Brasil), Cabo Verde, Córdoba (España) y Sevilla (España). En el análisis de resultados de este estudio se exponen con detalle los términos que asocian los futuros maestros y maestras a la inclusión. Si nos fijamos en la palabra que utilizan con mayor frecuencia en cada uno de estos lugares estudiados obtenemos el siguiente cuadro:

Tabla 1. Término más utilizado asociado a inclusión según cada país del estudio.

\begin{tabular}{|c|c|c|c|c|}
\hline & BRASIL & CABO VERDE & CÓRDOBA & SEVILLA \\
\hline IGUALDAD & $\mathrm{x}$ & $\mathrm{x}$ & & $\mathrm{x}$ \\
\hline INTEGRACIÓN & & & $\mathrm{x}$ & \\
\hline
\end{tabular}

Se puede observar cómo la palabra que ocupa el primer lugar en tres de las universidades estudiadas es «igualdad», seguida de la palabra «integración», que es priorizada por parte de una de las tres. Igualdad hace referencia a la tendencia a conseguir la equiparación de todos/as los/as ciudadanos/as en derechos y obligaciones. Por otra parte, la palabra integración hace referencia a la acción para que alguien o algo pase a formar parte de un todo. Un tipo de interpretación podría hacernos llegar a la conclusión de que los futuros docentes tienen la idea, el principio, hacia donde hay que tender: la igualdad de oportunidades para hacer sociedades más inclusivas. Pero, según nuestra interpretación, habría que hacer mucho más en las Facultades de Educación y Escuelas Normales de Magisterio para que esta ideología reconocida se convierta en competencias, y aprendizaje de estrategias con las que, en el futuro profesional en los centros educativos, sean capaces de conseguir escuelas inclusivas reales. Los autores concluyen que la inclusión educativa se entiende en los tres países estudiados como «un concepto que se nutre de ideas relativas a la igualdad, diversidad, integración, oportunidad, educación, respeto, ... esenciales para este término». (Lloret y Lloret, 2012, p.57).

Siguiendo a Payá (2010), podemos destacar como aspectos fundamentales sobre los que se debe centrar el aprendizaje reflexivo de los futuros docentes en relación con la inclusión, los siguientes:

a. Políticas, legislación y su aplicación en la práctica: En general, en todos los países, la legislación y la política educativa recogen los principios de las convenciones internacionales, tales como el reconocimiento de la educación como derecho humano fundamental, la no discriminación o el acceso universal a la educación. En muchas ocasiones el principal foco de atención es en la atención de personas con discapacidad. Sin embargo, la formación de docentes debe enfocarse como reflexión críticaque permita reconocer que una cosa es la legislación y otra el cumplimiento de esa legislación en la práctica. Reconocer las causas por las que esto ocurre debepriorizarse en la formación de docentes para la diversidad. El principal reto es conseguir una educación de calidad junto con equidad. En muchos escenarios es importante acompañar las medidas educativas de otras medidas dentro de planes integrales. Es importante destacar como un elemento clave de la Educación Inclusiva, potenciar la participación de la comunidad educativa y de la sociedad en general.

b. Diferentes concepciones de Educación Inclusiva: En América Latina pueden reconocerse, al menos, cuatro enfoques, según Payá (2010). Éstos son:

- Enfoque comprensivo o de Atención a la Diversidad: Dar respuesta a la diversidad en un marco común de Escuela, asegurando la igualdad de oportunidades, la lucha contra la discriminación y la promoción de la equidad. En esta línea se encuentran países como Argentina, Brasil, Ecuador, Honduras, Paraguay, Perú, Uruguay y Venezuela. En este aspecto comprensivo suele hablarse de currículo flexible y adaptado. 
- Enfoque que carece de planteamientos políticos claros o de prioridades en Educación Inclusiva. En esta línea pueden estar países como Bolivia o Guatemala.

- Enfoque de Educación Especial o de atención a alumnado con Necesidades Educativas Especiales. En esta línea se encuentra El Salvador.

- Enfoque doble de Educación Inclusiva y de atención a alumnado con Necesidades Educativas Especiales. En esta línea se encuentran países como Nicaragua.

c. Grupos prioritarios considerados: Según los países, las políticas suelen priorizar a: personas con discapacidad, alumnado con necesidades educativas especiales, grupos en riesgo de exclusión social o con gran vulnerabilidad, alumnado que abandonan los estudios, de procedencia rural, indígenas, no escolarizados, analfabetos, poblaciones desplazadas, mujeres, estudiantes con talento excepcional, niños hospitalizados, poblaciones afrodescendientes, grupos afectados por la violencia, sida, etc. (p.130).

d. Metodologías que atiendan la diversidad: Uso de metodologías, estrategias y adaptaciones que atiendan la diversidad de alumnado, generando climas de aula y de centro favorecedores de la inclusión.

Bronckbank y McGill (2002), proponen usar la práctica reflexiva como un medio, por el que se puede estimular a los estudiantes a que se observen a sí mismos y de mantener un diálogo crítico consigo mismos. El estudiante se hace preguntas sobre sus pensamientos y acciones.

Creemos que es importante que los futuros docentes, en su formación para la diversidad, tomen conciencia de los conocimientos prácticos al enfrentarlos con los teóricos, y reflexionen sobre las teorías, las prácticas y las incoherencias que se producen en la práctica, obstáculos y puntos positivos. Que tomen conciencia sobre el significado de sus experiencias y aprendizajes. Practicar mediante aprendizaje reflexivo «les permite a los estudiantes ser más conscientes de sus propios enfoques de aprendizaje».

El propósito de este trabajo es sugerir propuestas de mejora para que los futuros docentes, a lo largo de su formación inicial, obtengan un aprendizaje más reflexivo y profundo que permita realizar una práctica para la diversidad más adecuada en el marco de una educación inclusiva. Aunque las sugerencias que exponemos son muy útiles también para la formación del profesorado en ejercicio.

\section{MÉTODO}

Mediante una encuesta de preguntas abiertas pedimos a un grupo de estudiantes de México y de España que valoren cuál creen ellos que es la situación en las escuelas en relación con la inclusión. De esa manera se ponen en juego los conocimientos que obtienen en la formación inicial sobre inclusión educativa, las competencias que adquieren y su capacidad de comprensión del contexto escolar.

Esta encuesta cualitativa se realizó mediante una pregunta abierta al final de un cuestionario, que sirvió para averiguar la percepción que tenían los futuros docentes sobre la situación de la inclusión en los centros educativos, en el caso de España. En el caso de México se organizaron las principales respuestas obtenidas en España elaborando un conjunto de preguntas relacionadas con esas observaciones de los estudiantes españolas, pidiéndoles que escriban ejemplos y opiniones sobre esas cuestiones. Posteriormente, hemos analizado las respuestas a dichas preguntas y las hemos comparado. En total hemos trabajado con un grupo de clase de magisterio en España y otro grupo de clase en México, de unos 50 alumnos aproximadamente.

\section{RESULTADOS}

Hemos analizado las observaciones que indican tanto los estudiantes (futuros docentes) de España y de México, extrayendo conclusiones en forma de sugerencias para el aprendizaje reflexivo. Esta información puede ser de gran importancia para poder enriquecer los programas de asignaturas relacionadas con atención a la diversidad, tanto en México, como en España. Así como seminarios 
de capacitación para profesores en ejercicio. La información expuesta en la tabla puede ser usada como instrumento formativo de profesorado por lo que la exponemos completa a continuación:

Tabla 2. Comparación entre observaciones de estudiantes de Magisterio de España y de México. Sugerencias para mejorar su formación.

\begin{tabular}{|c|c|c|c|}
\hline Categoría & $\begin{array}{c}\text { Observaciones realizadas por los } \\
\text { estudiantes de España }\end{array}$ & $\begin{array}{l}\text { Observaciones realizadas por } \\
\text { los estudiantes de México }\end{array}$ & $\begin{array}{c}\text { Sugerencias para el } \\
\text { aprendizaje reflexivo en la } \\
\text { Formación de } \\
\text { docentes: MEJORAS. }\end{array}$ \\
\hline $\begin{array}{l}\text { 1.Altas Expectativas } \\
\text { para todos/as. }\end{array}$ & $\begin{array}{l}\text { El profesorado en ejercicio suele } \\
\text { poner altas expectativas de progreso } \\
\text { sólo en algunos alumnos. }\end{array}$ & $\begin{array}{l}\text { Se dejan llevar por cómo se } \\
\text { llevan con el alumno, más } \\
\text { que por el trabajo que hace. } \\
\text { Comparan a los alumnos. }\end{array}$ & $\begin{array}{l}\text { Es importante que las } \\
\text { altas expectativas se } \\
\text { tengan para todos. «No } \\
\text { pongamos límites». }\end{array}$ \\
\hline $\begin{array}{l}\text { 2.Educación } \\
\text { intercultural. }\end{array}$ & $\begin{array}{c}\text { En las aulas hay bastantes alumnos } \\
\text { de otros países: de países árabes, } \\
\text { de países africanos, ... }\end{array}$ & $\begin{array}{c}\text { Más que de culturas } \\
\text { diferentes, de contextos } \\
\text { diferentes. Hay diferentes } \\
\text { acentos y modismos. De } \\
\text { otros estados, usan palabras } \\
\text { desconocidas. }\end{array}$ & $\begin{array}{c}\text { Dedicar tiempo a } \\
\text { aprender estrategias para } \\
\text { la educación } \\
\text { intercultural (España) e } \\
\text { intercontextual (México). }\end{array}$ \\
\hline $\begin{array}{l}\text { 3.Formación en otras } \\
\text { necesidades } \\
\text { educativas } \\
\text { específicas que } \\
\text { suelen olvidarse. }\end{array}$ & $\begin{array}{l}\text { Hay niños con retraso de } \\
\text { aprendizaje, y con otras situaciones } \\
\text { relacionadas con ansiedad y demás, } \\
\text { además de otros alumnos con NEE. }\end{array}$ & $\begin{array}{l}\text { No hay muchos, } 1 \text { caso de } \\
\text { Cataratas, otro con } \\
\text { enfermedad desconocida. }\end{array}$ & $\begin{array}{c}\text { Debemos tener esto } \\
\text { presente y estar abiertos } \\
\text { a estudiar otras } \\
\text { necesidades específicas } \\
\text { que podrían presentarse } \\
\text { en el aula además de las } \\
\text { más comunes. }\end{array}$ \\
\hline $\begin{array}{l}\text { 4. Respeto para } \\
\text { todos/as. }\end{array}$ & $\begin{array}{l}\text { Hay maestros/as que tratan a todos } \\
\text { por igual aún habiendo } \\
\text { una gran diversidad. }\end{array}$ & $\begin{array}{l}\text { Tienen preferencias por algu- } \\
\text { nos. Con los que le } \\
\text { caen mal no. }\end{array}$ & $\begin{array}{l}\text { La clave de la inclusión } \\
\text { es tratar a todos con } \\
\text { igual respeto. }\end{array}$ \\
\hline $\begin{array}{l}\text { 5.Atención por igual } \\
\text { a todo el alumnado. }\end{array}$ & $\begin{array}{l}\text { Se suele dar preferencia a los } \\
\text { mejores alumnos. }\end{array}$ & $\begin{array}{l}\text { Tienen preferencia por algu- } \\
\text { nos, se refleja en las califica- } \\
\text { ciones, les dejan pasar prime- } \\
\text { ro, que elijan equipo. Genera } \\
\text { competitividad y malos } \\
\text { modos entre compañeros }\end{array}$ & $\begin{array}{l}\text { Como docentes debemos } \\
\text { tener la misma atención a } \\
\text { todo el alumnado. }\end{array}$ \\
\hline $\begin{array}{l}\text { 6. Usar un lenguaje } \\
\text { adecuado afectivo } \\
\text { con todo el } \\
\text { alumnado. }\end{array}$ & $\begin{array}{l}\text { Se suele regañar a los alumnos que } \\
\text { tienen más necesidades, incluso se } \\
\text { emplea un lenguaje algo despectivo. }\end{array}$ & $\begin{array}{l}\text { Sólo regañan cuando se dis- } \\
\text { traen. Pero la mayoría } \\
\text { de las veces no. }\end{array}$ & $\begin{array}{l}\text { Eliminar las prácticas } \\
\text { pedagógicas del regaño, } \\
\text { del castigo, del uso de un } \\
\text { lenguaje que despectivo. }\end{array}$ \\
\hline $\begin{array}{c}\text { 7.Sociedad / } \\
\text { Educación Inclusiva. }\end{array}$ & $\begin{array}{l}\text { La inclusión está muy atrasada. } \\
\text { Hasta que la sociedad no cambie su } \\
\text { manera de pensar y sus } \\
\text { comportamientos con la Escuela, } \\
\text { no cambiará la Educación. }\end{array}$ & $\begin{array}{l}\text { Está muy atrasada, muchas } \\
\text { personas no demuestran } \\
\text { respeto. Sigue existiendo la } \\
\text { discriminación. Muchos no } \\
\text { aceptan las diferencias, } \\
\text { incluso cuando son virtudes. } \\
\text { La sociedad tiene que estar } \\
\text { más informada. Falta } \\
\text { cultura de inclusión. }\end{array}$ & $\begin{array}{l}\text { Es preciso que avancen } \\
\text { tanto la sociedad como el } \\
\text { sistema educativo, pero } \\
\text { parece que la sociedad } \\
\text { es la que tiene que } \\
\text { cambiar más para dar } \\
\text { impulso a la educación. }\end{array}$ \\
\hline $\begin{array}{l}\text { 8.Atención a zonas } \\
\text { más vulnerables. }\end{array}$ & $\begin{array}{l}\text { En los centros que están en zonas } \\
\text { más desfavorecidas existe un mejor } \\
\text { clima de atención a la diversidad. }\end{array}$ & $\begin{array}{c}\text { Suele haber más exclusión } \\
\text { porque las personas tienen } \\
\text { diferentes tradiciones y for- } \\
\text { mas de pensar. }\end{array}$ & $\begin{array}{l}\text { Dedicar atención a com- } \\
\text { prender cómo proceder } \\
\text { ante la problemática } \\
\text { compleja y variada que se } \\
\text { presenta en las } \\
\text { zonas más }\end{array}$ \\
\hline
\end{tabular}




\begin{tabular}{|c|c|c|c|}
\hline $\begin{array}{l}\text { 9.Un buen trato } \\
\text { (afectivo y } \\
\text { pedagógico). }\end{array}$ & $\begin{array}{l}\text { Cuando un niño con discapaci- } \\
\text { dad es tratado de buena forma, } \\
\text { el aprendizaje se produce } \\
\text { mejor. Esto ocurre por ejemplo } \\
\text { con alumnado con síndrome de } \\
\text { Asperger. «Si se capta la aten- } \\
\text { ción del alumnado con NEE } \\
\text { todo es posible» }\end{array}$ & $\begin{array}{c}\text { Por lo general se les brinda } \\
\text { mayores } \\
\text { atenciones. Se le da segui- } \\
\text { miento pero no se trata. A } \\
\text { los niños con síndrome de } \\
\text { Down se les trata bien.Se } \\
\text { les da afecto especial y se } \\
\text { les ayuda. }\end{array}$ & $\begin{array}{c}\text { A veces es tan sencillo como } \\
\text { tratar adecuadamente al } \\
\text { alumnado y el aprendizaje, el } \\
\text { buen comportamiento, etc. va } \\
\text { viniendo. }\end{array}$ \\
\hline $\begin{array}{l}\text { 10. El apoyo dentro } \\
\text { del aula ordinaria. }\end{array}$ & $\begin{array}{l}\text { Cuando sacan a un alumno de } \\
\text { clase para llevarlo al aula de } \\
\text { Educación Especial, el alumno } \\
\text { se siente mal. }\end{array}$ & $\begin{array}{l}\text { En un caso de audición lo } \\
\text { sacaban pero lo integraron } \\
\text { bien en el salón de clases. } \\
\text { Se sienten mal porque en } \\
\text { ese momento todos lo ob- } \\
\text { servan e incluso lo señalan } \\
\text { y él cree que es el } \\
\text { peor del mundo. }\end{array}$ & $\begin{array}{l}\text { El apoyo debe } \\
\text { producirse } \\
\text { dentro del aula. }\end{array}$ \\
\hline $\begin{array}{l}\text { 11. Aprendizaje } \\
\text { cooperativo mejora la } \\
\text { autoestima. }\end{array}$ & $\begin{array}{l}\text { Cuando no se tiene en cuenta al } \\
\text { alumnado con NEE le afecta a } \\
\text { la autoestima y a la relación con } \\
\text { los compañeros. }\end{array}$ & $\begin{array}{l}\text { Cuando se le ignora se } \\
\text { le daña su autoestima o } \\
\text { reciben burlas a su persona. } \\
\text { También cuando no se le } \\
\text { deja participar y levanta la } \\
\text { mano. Cuando no sele tiene } \\
\text { en cuenta. }\end{array}$ & $\begin{array}{c}\text { Se debe fomentar la interacción } \\
\text { entre compañeros mediante } \\
\text { las estrategias cooperativas } \\
\text { adecuadas. }\end{array}$ \\
\hline 12. Dar ejemplo & $\begin{array}{l}\text { Si un profesor trata con respeto } \\
\text { a todos, los compañeros del } \\
\text { alumno con NEE, también lo } \\
\text { hacen. Si ocurre al contrario, los } \\
\text { compañeros le } \\
\text { imitan también. }\end{array}$ & $\begin{array}{l}\text { Se suele copiar lo que el } \\
\text { profesor hace. Si el profe- } \\
\text { sor trata bien a la niña con } \\
\text { síndrome de Down los } \\
\text { demás le imitan. }\end{array}$ & $\begin{array}{l}\text { Tomar conciencia de la } \\
\text { enorme influencia que tiene } \\
\text { nuestro comportamiento } \\
\text { como docentes con una } \\
\text { buena actitud y tratamiento } \\
\text { de todos/as. }\end{array}$ \\
\hline $\begin{array}{l}\text { 13. Colaboración } \\
\text { de las instituciones } \\
\text { locales. }\end{array}$ & $\begin{array}{l}\text { Las instituciones locales no } \\
\text { suelen colaborar con el centro } \\
\text { en actividades educativas. }\end{array}$ & No se conoce. & $\begin{array}{l}\text { Una clave de la inclusión está } \\
\text { relacionada con el nivel de } \\
\text { involucramiento en el centro } \\
\text { de las instituciones locales. }\end{array}$ \\
\hline $\begin{array}{l}\text { 14. La innovación } \\
\text { docente para la } \\
\text { inclusión. }\end{array}$ & $\begin{array}{l}\text { Siempre tenemos que innovar } \\
\text { como docentes. }\end{array}$ & $\begin{array}{l}\text { Se deben llevar dinámicas } \\
\text { cuando hay algunos con } \\
\text { NEE. Con la inclusión el } \\
\text { profesorado debe abrirse a } \\
\text { un cambio. Debe salirse de } \\
\text { lo tradicional en cuanto a } \\
\text { castigos. Debemos prepa- } \\
\text { rarnos para usar estrategias } \\
\text { inclusivas. Siempre debe } \\
\text { estar innovando para } \\
\text { adaptarse a las distintas } \\
\text { situaciones. }\end{array}$ & $\begin{array}{l}\text { La innovación docente tiene } \\
\text { mucho que ver con la atención } \\
\text { a la diversidad puesto que la } \\
\text { utilización de estrategias inno- } \\
\text { vadoras puede dar respuesta } \\
\text { a problemas a veces difíciles. }\end{array}$ \\
\hline $\begin{array}{l}\text { 15. Formación del } \\
\text { profesorado en } \\
\text { ejercicio. }\end{array}$ & $\begin{array}{l}\text { No todos los docentes están } \\
\text { suficientemente formados ni } \\
\text { tienen una actitud adecuada. }\end{array}$ & $\begin{array}{c}\text { No todos están bien } \\
\text { formados. Algunos no se } \\
\text { abren al cambio. Otros no } \\
\text { van y luego ponen 10. Falta } \\
\text { mucha preparación para la } \\
\text { inclusión. Muchos no } \\
\text { tuvieron una práctica. }\end{array}$ & $\begin{array}{c}\text { La diversidad de } \\
\text { profesorado no está } \\
\text { suficientemente atendida. } \\
\text { Sin embargo deben tener un } \\
\text { denominador común: la actitud } \\
\text { positiva hacia la inclusión. La } \\
\text { educación es un derecho de } \\
\text { todas las personas. }\end{array}$ \\
\hline
\end{tabular}




\begin{tabular}{|c|c|c|c|}
\hline $\begin{array}{l}\text { 16. Coordinación entre } \\
\text { agentes implicados. }\end{array}$ & $\begin{array}{l}\text { Se tiene que conseguir una } \\
\text { cooperación entre } \\
\text { familias y profesorado. }\end{array}$ & $\begin{array}{l}\text { Entre profesores y familia- } \\
\text { res sí hay buena relación. } \\
\text { Tienen que formar un buen } \\
\text { equipo para trabajar. }\end{array}$ & $\begin{array}{l}\text { La coordinación y colabora- } \\
\text { ción tiene que ser muy buena } \\
\text { entre alumnado, profesorado, } \\
\text { familias e instituciones. }\end{array}$ \\
\hline $\begin{array}{l}\text { 17. El nivel de inclusión } \\
\text { es bajo. }\end{array}$ & $\begin{array}{l}\text { Todavía queda mucho que } \\
\text { mejorar para que la } \\
\text { Educación Inclusiva sea } \\
\text { una realidad. }\end{array}$ & $\begin{array}{l}\text { Hay un nivel medio, unos lo } \\
\text { llevan y otros no. Más bien } \\
\text { bajo. Hay altos índices de } \\
\text { discriminación. No hay } \\
\text { cultura de inclusión } \\
\text { en la sociedad. }\end{array}$ & $\begin{array}{l}\text { La percepción en general } \\
\text { es que se ha mejorado algo } \\
\text { pero aún queda mucho } \\
\text { por hacer. }\end{array}$ \\
\hline $\begin{array}{l}\text { 18. La importancia de la } \\
\text { implicación y motivación } \\
\text { del profesorado. }\end{array}$ & $\begin{array}{l}\text { La implicación del profesora- } \\
\text { do puede hacer que funcionen } \\
\text { situaciones incluso con pocos } \\
\text { recursos. }\end{array}$ & $\begin{array}{l}\text { Con buena actitud y motiva- } \\
\text { ción hay buenos resultados } \\
\text { y se resuelven los obstácu- } \\
\text { los. Que tenga seguridad. }\end{array}$ & $\begin{array}{l}\text { La gran motivación e implica- } \\
\text { ción del profesorado puede } \\
\text { incluso compensar la } \\
\text { falta de otros recursos. }\end{array}$ \\
\hline 19. Nivel de inclusión & $\begin{array}{l}\text { Haciendo una estimación, } \\
\text { en apariencia podría } \\
\text { hablarse de un } 90-95 \% \\
\text { del nivel de inclusión } \\
\text { alcanzado, cuando en la } \\
\text { realidad es de sólo el } \\
45-55 \% \text {. Pudiera situarse } \\
\text { en un nivel medio-bajo. }\end{array}$ & $\begin{array}{l}60 \text { - } 65 \% \text {. En algún } \\
\text { caso se señala el } 30 \% \text {. } \\
\text { Lo que nos acercaría si } \\
\text { hicíéramos media al } 45 \% \\
\text { aproximadamente. }\end{array}$ & $\begin{array}{c}\text { Es importante reflexionar } \\
\text { acerca del nivel alcanzado } \\
\text { en inclusión a la vista de } \\
\text { los indicios. }\end{array}$ \\
\hline $\begin{array}{l}\text { 20. Concienciación } \\
\text { a la sociedad y } \\
\text { al profesorado. }\end{array}$ & $\begin{array}{l}\text { Existen todavía } \\
\text { muchos prejuicios. }\end{array}$ & $\begin{array}{l}\text { Existen muchos prejui- } \\
\text { cios como la forma de } \\
\text { vestir, la sexualidad, los } \\
\text { discapacitados. }\end{array}$ & $\begin{array}{l}\text { La tradición ha dejado en } \\
\text { nuestra conciencia colecti- } \\
\text { va muchos temores, mitos } \\
\text { y prejuicios que conviene } \\
\text { identificar para establecer } \\
\text { las ideas correctas. }\end{array}$ \\
\hline $\begin{array}{l}\text { 21. Misma atención } \\
\text { como profesionales a } \\
\text { todas las NEE. }\end{array}$ & $\begin{array}{l}\text { Hay veces en que se observa } \\
\text { que a un determinado tipo de } \\
\text { NEE se le da diferente aten- } \\
\text { ción, por ejemplo a un niño } \\
\text { con síndrome de Down } \\
\text { mejor que a otro con } \\
\text { síndrome de Asperger, ... }\end{array}$ & $\begin{array}{l}\text { No se han observado casos. } \\
\text { Cada NEE requiere trata- } \\
\text { mientos diferentes según } \\
\text { sus necesidades, aunque se } \\
\text { les debe tratar con la } \\
\text { misma atención o interés. }\end{array}$ & $\begin{array}{l}\text { Las mayores dificultades } \\
\text { podrían hacer que disminu- } \\
\text { ya nuestra atención como } \\
\text { profesionales y búsqueda de } \\
\text { estrategias. Se debe com- } \\
\text { pensar esta situación. }\end{array}$ \\
\hline $\begin{array}{l}\text { 22. El máximo de la } \\
\text { inclusión. }\end{array}$ & $\begin{array}{l}\text { El máximo de la inclusión, si } \\
\text { hiciéramos una prospectiva } \\
\text { es que se trabaje de manera } \\
\text { normal, natural con todos. }\end{array}$ & $\begin{array}{l}\text { Que todos sean iguales. } \\
\text { Cambiaría radicalmente la } \\
\text { forma de enseñar. Se ayuda- } \\
\text { ría a aceptar las diferencias. }\end{array}$ & $\begin{array}{c}\text { La inclusión, en su perfec- } \\
\text { ción sería una escolarización } \\
\text { normal, diversificada pero } \\
\text { natural y normal donde cada } \\
\text { uno puede desarrollar sus } \\
\text { potencialidades. }\end{array}$ \\
\hline $\begin{array}{l}\text { 23. Discriminación positi- } \\
\text { va (o Acción positiva). }\end{array}$ & $\begin{array}{l}\text { Tratar a todos por igual, } \\
\text { también es un error. }\end{array}$ & $\begin{array}{l}\text { Todos tenemos los mismos } \\
\text { derechos. Todos merecemos } \\
\text { respeto pero adaptándonos } \\
\text { a los diferentes comporta- } \\
\text { mientos. Hay que ser equi- } \\
\text { tativo. Mismos derechos con } \\
\text { diferentes características. }\end{array}$ & $\begin{array}{l}\text { Tratar a todos por igual como } \\
\text { si no tuvieran discapacida- } \\
\text { des, sin hacer distinciones } \\
\text { está mal, hay que ayudar a } \\
\text { cada cual según sus } \\
\text { necesidades. Dar el apoyo } \\
\text { que necesita cada uno. }\end{array}$ \\
\hline
\end{tabular}




\begin{tabular}{|c|c|c|c|}
\hline 24. Resaltar lo positivo. & $\begin{array}{c}\text { Algunos profesores resaltan } \\
\text { las necesidades y } \\
\text { discapacidades delante de } \\
\text { los demás y eso } \\
\text { perjudica a la autoestima. }\end{array}$ & $\begin{array}{l}\text { Los profesores resaltan las } \\
\text { discapacidades, a veces lo } \\
\text { hacen. Y eso afecta al com- } \\
\text { portamiento. Se } \\
\text { sienten menos. }\end{array}$ & $\begin{array}{l}\text { Es preciso no resaltar las } \\
\text { discapacidades y dificulta- } \\
\text { des delante de los } \\
\text { compañeros porque genera } \\
\text { un clima de rechazo y } \\
\text { afecta a la autoestima del } \\
\text { alumno con NEE. Esto } \\
\text { hace que los demás lo vean } \\
\text { como inaccesible y diferente } \\
\text { en sentido negativo. Se } \\
\text { deben resaltar las } \\
\text { cualidades, lo positivo. }\end{array}$ \\
\hline 25. Voluntariado en el aula. & $\begin{array}{l}\text { Los voluntarios ayudan } \\
\text { muchísimo y generan un } \\
\text { buen clima inclusivo y de } \\
\text { colaboración entre todos. }\end{array}$ & $\begin{array}{l}\text { Es buena idea que permitan } \\
\text { a voluntarios/as ayudar en } \\
\text { las Escuelas. Sería de gran } \\
\text { utilidad. Por ejemplo a } \\
\text { través de la oficina de } \\
\text { universidad y desarrollo. }\end{array}$ & $\begin{array}{c}\text { El voluntariado debe } \\
\text { integrarse en las tareas } \\
\text { cooperativas del aula, esto } \\
\text { supone un beneficio para } \\
\text { todo el alumnado, al mismo } \\
\text { tiempo que una ayuda para } \\
\text { el tutor. }\end{array}$ \\
\hline $\begin{array}{l}\text { 26. Prácticas de } \\
\text { anticipación. }\end{array}$ & $\begin{array}{l}\text { Es posible que el } \\
\text { profesorado que no lo hace } \\
\text { bien le falte experiencia o } \\
\text { formación o interés. }\end{array}$ & $\begin{array}{l}\text { Los profesores tienen que } \\
\text { informarse más sobre } \\
\text { inclusión. No fueron } \\
\text { formados. En algunos } \\
\text { casos es falta de actitud. }\end{array}$ & $\begin{array}{l}\text { La falta de experiencia } \\
\text { se puede suplir con más } \\
\text { prácticas y lo que es más } \\
\text { importante con experiencias } \\
\text { prácticas de anticipación, } \\
\text { que enriquezcan el } \\
\text { número de prácticas. }\end{array}$ \\
\hline $\begin{array}{l}\text { 27. La inclusión son opor- } \\
\text { tunidades de aprendizaje } \\
\text { para todos. }\end{array}$ & $\begin{array}{l}\text { Profundizando en el por } \\
\text { qué ocurren prácticas poco } \\
\text { inclusivas puede ser porque } \\
\text { el profesorado lo vive como } \\
\text { un problema y no } \\
\text { como una ventaja. }\end{array}$ & $\begin{array}{c}\text { En algunos casos puede ser } \\
\text { que lo vivan } \\
\text { como un problema. }\end{array}$ & $\begin{array}{l}\text { La inclusión es una } \\
\text { oportunidad para } \\
\text { enriquecerse personal y } \\
\text { profesionalmente. Se dan } \\
\text { oportunidades de } \\
\text { aprendizaje para todos, } \\
\text { incluso para ser } \\
\text { mejores profesores. }\end{array}$ \\
\hline
\end{tabular}

\section{DISCUSIÓN}

Tal y como dice uno de los futuros docentes que respondió a nuestra pregunta: A día de hoy es cierto que la inclusión no está del todo ligada a la educación tal y como debería, pero con el paso del tiempo y con la máxima implicación de familia y escuela podemos hacer que esto sea posible.

Podemos sintetizar diciendo que en relación a la inclusión, según nuestros datos, que en España se está intentado conseguir en las escuelas, aunque se aprecia que aún queda mucho por hacer. Nuestros datos señalan alrededor de un $45-55 \%$ el nivel de consecución de la inclusión, aunque tal vez pueda arriesgarse un porcentaje de entre 50 y $60 \%$.

Por su parte en México la situación, puede tal vez, definirse como caótica, necesitando establecer una coordinación y un orden entre la legislación y las políticas, la formación de docentes para atender la diversidad, las prácticas inclusivas y la concienciación social.

La inclusión real es aún un sueño, un esperado final feliz para todos esos alumnos que ejercieron su derecho a graduarse, pero no su derecho a participar y a aprender en las escuelas. (Sánchez, 2016). Aún así se sitúa en torno al 45\%, como aproximación de nuestros datos. 
Según la autora, en México existen dos unidades que no siempre consiguen que el alumnado con NEE consigan la inclusión. Se trata de los Centros de Atención Múltiple (CAM) como servicio escolarizado para alumnos en condición de discapacidad o trastornos graves del desarrollo, y en las Unidades de Apoyo a la Escuela Regular (USAER). Un USAER es una célula de especialistas que se encuentran dentro de las escuelas trabajando en colaboración con los maestros de grupo. Tal vez pueden servir los siguientes datos para ilustrar superficialmente la situación en México: hay 450 centros para atención para estudiantes discapacitados en educación media superior. En educación básica se calcula que hay 300 mil niños con discapacidad; $24.8 \%$ de las personas con discapacidad no saben leer y escribir; solo $45 \%$ en edad escolar asiste a clases y el promedio de escolaridad es de 4.8 años (UNOiNews,2016).

Por su parte, Ramírez Moguel (s.f.) dice que:

Aunque la inclusión educativa está muy lejos de ser una realidad en la mayoría de las escuelas del país, es importante reconocer que ha sido parte de un proceso en donde se han observado cambios importantes.

\section{CONCLUSIONES}

La clave está en la actitud, compromiso, implicación y motivación del profesorado. Tanto del profesorado novel como del experto. Por lo tanto el riesgo es que a pesar de una formación de calidad y en la línea de lo que en este trabajo se está planteando, el profesorado se quede en lo superficial y no lleguen a profundizar en lo esencial. Y lo que es peor que se dejen influenciar por las actitudes superficiales que enfrentan la escasez de recursos, humanos y materiales y otras situaciones a la verdadera tarea de hacer una inclusión efectiva. A la que tienen derecho todos los alumnos/as.

\section{REFERENCIAS BIBLIOGRÁFICAS}

Anijovich, R. et al (2009). Transitar la formación pedagógica. Buenos Aires, Argentina: Paidós.

Llorent, V. y Llorent, V. J. (2012). La inclusion educativa desde la perspectiva de futuros maestros en Brasil, Cabo Verde y España. Revista Educación Inclusiva, vol. 5, №2, p.p. 43-59.

Payá, A. (2010). Políticas de Educación Inclusiva en América Latina. Propuestas, realidades y retos de future. Revista de Educación Inclusiva, vol. 2, N. ${ }^{\circ} 3$.

Ramírez Moguel (s.f.). Reflexión sobre la inclusión educativa en México y los avances para estudiantes con discapacidad múltiple. Perkins. Recuperado de: http://www.perkinsla.org/recursosbiblio/153_1Educacion\%20Inclusiva\%20-\%20 M\%20Ramirez.pdf.

Sánchez, T. (2016). Retos de la inclusión educativa en México. Recuperado de: https://www.animalpolitico.com/blogueros-aprender-es-mi-derecho/2016/10/03/retos-la-inclusion-educativa-mexico/.

UNOiNews (2016). Los retos de la Educación Inclusiva en México. Recuperado de: http://mx.unoi.com/2016/09/13/los-retos-de-la-inclusion-educativa-en-mexico/. 